\title{
Bias in Uncontrolled Brain Tumor Trials
}

Malignant gliomas are extremely difficult to treat. Even after aggressive multimodality therapy, the median survival for the most malignant tumor glioblastoma multiforme (GBM) is about a year, and for the slightly less malignant anaplastic astrocytoma, perhaps 18 months. ${ }^{1}$ While these survival figures are better than those reported 30 years ago, they are still bleak. The one encouraging aspect in the treatment of this disease has been the extraordinary growth of the number of research programs devoted to improving treatment. The research "growth industry" includes both laboratory and clinical endeavors. Results from the laboratory have occasionally bordered on the spectacular for example, the identification of the importance of the tumor suppressor gene $\mathrm{p} 53$, and how research into cellular heterogeneity has led to better understanding of cellular genetics in tumor progression. The clinical therapy trials may be characterized as slow, plodding and at best marginally successful. Especially problematic is the difficulty of converting laboratory discoveries into clinical successes.

Nevertheless, literally hundreds of clinical trials of brain tumor therapy have been conducted, by far the largest number being uncontrolled Phase II studies. Often these studies yield exciting results - treated patients living two or three times longer than "historical controls". The excitement wanes when the "wonder" drug or technique is subject to a subsequent controlled trial and the treated patients fair no better (and sometimes worse) than the concurrent control patients. Why is it that what seems so good in Phase II trial turns out to be useless in a controlled trial? One very real answer is that the Phase II trial inadvertently selects patients destined to live longer anyway, whether or not they received the wonder treatment. This form of bias in uncontrolled trials has been the subject of several studies, some of the best of which performed by Cairncross and his colleagues. ${ }^{2,3}$ They have suggested that uncontrolled interstitial (brachytherapy) trials suffer such bias, as do uncontrolled radiosurgery trials. Their most recent effort, the study reported in this issue, ${ }^{4}$ addresses the radiosurgery issue. How well they do and how valid are their conclusions, are the subjects of this editorial.

Irish et al. present a thoughtful analysis of a model for stereotactic radiosurgery using their population-based data base ${ }^{3}$ to "match" that of Loeffler et al. who treated patients with radiosurgery in an uncontrolled trial. 5,6 Using the same eligibility criteria reported by Loeffler et al. Irish et al. selected similar patients from their database, and compared the outcome of standard treatment of these patients to that of ineligible patients also drawn from their database. The two most important eligibility criteria were tumor size ( $\leq 4 \mathrm{~cm}$ maximum diameter on postradiation scan) and Karnofsky performance status $(\geq 70)$. Patients in the database meeting these criteria but treated in conventional fashion (i.e., without radiosurgery), lived significantly longer than did those not eligible. The authors consider it highly likely that the reason radiosurgery looks so good in the studies of Loeffler et al. is because particularly "good" patients were selected; patients with small tumors and Karnofsky scores of at least 70 are likely to do well no matter how they are treated. The study by Irish et al. is well done and the results persuasive. Some of their conclusions, however, are less persuasive, at least to this reviewer.

Irish et al. note that radiosurgery-treated patients in Loeffler's study "lived slightly longer" than patients in the radiosurgery-eligible control group culled from the Irish et al. database (19.7 vs. 16.4 months) [italics added]. The judgmental term "slightly longer" referring to the median survival might well translate into a quite prolonged tail group of $20-30 \%$ of patients surviving two or more years. Absent a comparison of survival curves it is not possible to tell. Irish et al. then proceed to list a number of biases that might have been introduced to account for even this modest difference. However, they have no way of modeling these additional factors and appear to offer them primarily to support their own argument. Is this perhaps a bit of their own "bias"?

Then Irish et al. "question whether a randomized controlled trial is truly necessary" since "a phase III study of stereotactic radiosurgery as currently prescribed for malignant glioma is unlikely to yield a positive result". By the last assertion, I presume is meant "yield a result indicating that radiosurgery plus standard therapy is better than standard therapy alone". They concede that their analysis has yet to establish itself as having predictive credentials, but allude to trials in intraartial chemotherapy and interstitial radiation to support their views. It should be pointed out that the Radiation Therapy Oncology Group (RTOG) randomized Phase III trial of stereotactic radiosurgery has broadened the eligibility to include patients with Karnofsky of 60 or better, thus making the results more widely applicable. ${ }^{7}$

It seems to me that Irish et al. can't have it both ways. Their analysis beautifully demonstrates that uncontrolled Phase II trials draw conclusions that are highly likely to be invalid because of biases introduced into selecting patients for the study. They then conclude that controlled trials may not work either because of other biases, which, in fact, they did not analyze. Polemics aside, the real problem is that Phase II uncontrolled trials use patients, resources and time to yield results which, if positive according to the investigators, need to be tested in "Phase III controlled trials". Indeed that is precisely the conclusion of Loeffler et al. about stereotactic radiosurgery.

Examples abound of positive Phase II trials recommending "follow-up randomized studies". Out of the hundreds of Phase II glioma chemotherapy trials documented so well by Mahaley, ${ }^{8}$ how many drugs were tested in controlled trials to find their way into the clinic? Cooperative group trials, e.g., by the 
Brain Tumor Study Group (BTSG)/Brain Tumor Cooperative Group (BTCG), RTOG, Southwest Oncology Group (SWOG), European Organization for Research and Treatment of Cancer (EORTC) and others have identified few truly effective drugs. However, 1,3-bis(2-chloroethyl)-1-nitrosourea (carmustine) (BCNU), procarbazine and methyl-1(2-chloroethyl(-3-cyclohexyl-1-nitrosourea (methyl-CCNU) were better than placebo, ${ }^{9}$ and the first two are part of the standard chemotherapy for gliomas. The most popular combination, procarbazine, 1(2chloroethyl)-3-cyclohexyl-1-nitrosourea (lomustine(CCNU), vincristine (PCV), was no better than BCNU when tested in a controlled trial, ${ }^{10}$ but was probably better for anaplastic astrocytomas per se." Its use for anaplastic oligodendroglioma is currently undergoing controlled testing. Equally important, a number of drugs touted to be useful based on Phase II trials, were found to have little value when tested in controlled fashion.

If I may be permitted a radical idea, except for dose-seeking trials or those for toxicity, perhaps all glioma therapy trials should be controlled. If we agree that only by prospective randomized trials can improvement in therapy of gliomas be substantiated, then perhaps we would be better off performing such trials right from the beginning. The primary requisite of such programs is that an adequate number of patients be accrued to complete the trials in a timely fashion. Assuring such accrual will likely require cooperative programs among several centers.

The history of the treatment of malignant gliomas includes numerous instances in which the promise of an advance in treatment held out in a Phase II trial was not confirmed in a Phase III trial. Three examples come to mind. In one, interstitial radiotherapy was touted as being effective for malignant gliomas. ${ }^{12}$ As noted by Irish et al. patient selection clearly influenced this result. The BTCG I-125 interstitial radiation controlled trial found only modest benefit. ${ }^{13}$ Another example was suggested by a Phase II study in which hyperfractionated radiotherapy was declared more effective for brain stem glioma than single daily dosing ${ }^{14}$ Now, several years later, and after many trials ${ }^{15-17}$ it is clear that hyperfractionation is no better. The third example is intraarterial BCNU chemotherapy, in which many Phase II trials suggested that the intraarterial route for $\mathrm{BCNU}$ was both safe and effective. A BTCG randomized controlled trial proved both assertions wrong.$^{18}$ How much money was spent and how many patients were accrued in the original Phase II trials for these treatments? What would have been the outcome if each had been done as a controlled trial from the outset? I for one, think the answer is that money, time and patients would have been saved by such an approach.

Irish et al. have performed an invaluable service by pointing out just how bias in uncontrolled trials leads to incorrect conclusions about the treatment under investigation. To my mind, their analysis supports the notion that the most ethical studies are those that lead to definitive conclusions. The most unethical trial is one that uses patients without yielding results that precisely define an end-point which helps other patients. Controlled trials that provide definitive conclusions may be the only useful and, therefore, the only ethical trials.

William R. Shapiro Phoenix, Arizona, USA

\section{REFERENCES}

1. Shapiro WR, Shapiro JR, Walker RW. Management of specific malignancies: Central nervous system. In: Abeloff MD, Armitage JO, Lichter AS, Niederhuber JE, eds. Clinical Oncology. New York: Churchill Livingston, 1995: 851-912.

2. Winger MJ, Macdonald DR, Schold SC Jr, Cairncross JG. Selection bias in clinical trials of anaplastic glioma. Ann Neurol 1989; 26: 531-534.

3. Florell RC, Macdonald DR, Irish WD, et al. Selection bias, survival, and brachytherapy for glioma. J Neurosurg 1992; 76: 179183.

4. Irish WD, Macdonald DR, Cairncross JG. Measuring bias in uncontrolled brain tumor trials: to randomize or not to randomize? Can J Neurol Sci 1997; 24: 307.

5. Loeffler JS, Alexander E III, Shea WM, et al. Radiosurgery as part of the initial management of patients with malignant gliomas. J Clin Oncol 1992; 10: 1379-1385.

6. Loeffler JS, Shrieve DC, Wen PY, et al. Radiosurgery for intracranial malignancies. Sem Radiat Oncol 1995; 3: 225-234.

7. Loeffler JS, Shrieve DC, Alexander E III. Radiosurgery for glioblastoma multiforme: the importance of selection criteria. Int J Radiat Oncol Biol Phys 1994; 30: 731-733.

8. Mahaley MS. Neuro-oncology index and review (adult primary brain tumors). Radiotherapy, chemotherapy, immunotherapy, photodynamic therapy. J Neurooncol 1991; 11: 85-147.

9. Green SB, Byar DP, Walker MD, et al. Comparisons of carmustine, procarbazine and high-dose methylprednisolone as additions to surgery and radiotherapy for the treatment of malignant glioma. Cancer Treat Rep 1983; 67: 121-132.

10. Levin VA, Wara WM, Davis RL, et al. Phase III comparison of BCNU and the combination of procarbazine, CCNU, and vincristine administered after radiotherapy with hydorxyurea for malignant gliomas. J Neurosurg 1985; 63: 218-223.

11. Levin VA, Silver P, Hannigan J, et al. Superiority of post-radiotherapy adjuvant chemotherapy with CCNU, procarbazine, and vincristine (PCV) over BCNU for anaplastic gliomas: NCOG 6G61 final report. Int J Radiat Oncol Biol Phys 1990; 18: 321-324.

12. Prados MD, Gutin PH, Phillips TL, et al. Interstitial brachytherapy for newly diagnosed patients with malignant gliomas: the UCSF experience. Int J Radiat Oncol Biol Phys 1992; 24: 593-597.

13. Shapiro WR, Green SB, Burger P, et al. A randomized trial of interstitial radiotherapy (IRT) boost for the treatment of newly diagnosed malignant glioma (glioblastoma multiforme, anaplastic astrocytoma, anaplastic oligodendroglioma, malignant mixed glioma): BTCG study 8701. Neurology 1994; 44 (Suppl 2): 263 (Abstract).

14. Edwards MSB, Wara WM, Urtasun RC, et al. Hyperfractionated radiation for brain-stem glioma: a Phase I-II trial. J Neurosurg 1989; 70: 691-700.

15. Packer RJ, Boyett JM, Zimmerman RA, et al. Hyperfractionated radiation therapy ( $72 \mathrm{~Gy}$ ) for children with brain stem gliomas: a Childrens Cancer Group phase I/II Trial. Cancer 1993; 72: 14141421.

16. Freeman CR, Krischer JP, Sanford RA, et al. Final results of a study of escalating doses of hyperfractionated radiotherapy in brain stem tumors in children: a Pediatric Oncology Group study. Int J Radiat Oncol Biol Phys 1993; 27: 197-206.

17. Packer RJ, Boyett JM, Zimmerman RA, et al. Outcome of children with brain stem gliomas after treatment with $7800 \mathrm{cGy}$ of hyperfractionated radiotherapy: a Childrens Cancer Group Phase I/II Trial. Cancer 1994; 74: 1827-1834.

18. Shapiro WR, Green SB, Burger PC, et al. A randomized comparison of intra-arterial versus intravenous BCNU, with or without intravenous 5-fluorouracil, for newly diagnosed patients with malignant glioma. J Neurosurg 1992; 76: 772-781. 